American Journal of Applied Sciences 7 (8): 1139-1147, 2010

ISSN 1546-9239

(C) 2010 Science Publications

\title{
Link Stability and Node Energy Conscious Local Route-Repair Scheme for Mobile Ad Hoc Networks
}

\author{
${ }^{1}$ Anuradha Banerjee and ${ }^{2}$ Paramartha Dutta \\ ${ }^{1}$ Department of Computer Applications, Kalyani Government Engineering College, \\ Kalyani, Nadia, West Bengal, India \\ ${ }^{2}$ Department of Computers and Systems Science, Visva-Bharati University, \\ Santiniketan, West Bengal, India
}

\begin{abstract}
Problem statement: A mobile ad hoc network is a self-configuring network of mobile nodes connected by wireless links. As the nodes are free to move randomly, topology of the network may change rapidly and unpredictably. As a result, intermediate nodes in the communication routes tend to lose connection with each other during the communication process. In order to repair a broken link, an end-toend (from source to destination) route discovery is typically performed to establish a new route for communication. The mechanism results in huge communication overhead and long delay in completion of the communication. So, it is rational to locally repair a link, as much as possible, than to discover a whole new route. Approach: In the present article, we proposed a Link Stability and Node Energy Conscious (LSNEC) local route repair scheme for mobile ad hoc networks. In case of breakage of a link from node $n_{a}$ to another node $n_{b}$ in between a communication session, LSNEC instructs $n_{a}$ to broadcast a ROUTEREPAIR message within its radio-range specifying $n_{b}$ or any successor of $n_{b}$ in the broken route, as a desirable destination. If any node residing within the radio-range of $n_{a}$ has an already established single/multi-hop path to any one of those destinations mentioned in the ROUTE-REPAIR message, it sends a REPAIR-ACK message back to $n_{\mathrm{a}}$. Attributes of REPAIR-ACK include the identification number (s) of the destination (s) as well as identification numbers, geographical positions (in terms of latitude and longitude), radio-ranges and residual energies of nodes present in the associated path to that destination along with the intuitively computed stability of links between the consecutive nodes. The stability is computed depending upon relative velocities of the involved nodes, distance between them and the history of survival of the link. The optimal path is chosen depending upon the residual energy of nodes, stability of links and number of hops in that path. Results: In simulations we compared the LSNEC embedded versions of the protocols Ad hoc On-demand Distance Vector routing (AODV) and Associatively Based Routing (ABR) with their ordinary versions as well as PATCH and "Quick Local Repair" scheme (to be referred to as QLR subsequently in this study) embedded versions. Both PATCH and QLR are wellknown local repairing schemes for ad hoc networks. It is found that, LSNEC-AODV dramatically saves $57 \%$ control overhead compared to ordinary AODV, 32.7\% compared to PATCH-AODV and 36.3\% compared to QLR-AODV. Similarly, LSNEC-ABR saves $29.3 \%$ control overhead than ordinary ABR, $10.7 \%$ than PATCH-ABR and $12.8 \%$ than QLR-ABR. Accordingly decrease the power consumption of network nodes and delay in recovery. Conclusion: The simulation results emphatically illustrated the performance benefit of our proposed scheme LSNEC compared to the other state-of-the-art local routerepair schemes, in respect of control overhead, overall energy consumption of the network, data packet delivery ratio and route recovery delay. LSNEC has its application wherever an ad hoc network is used. Please note that ad hoc networks are extremely useful in military and other tactical applications such as emergency rescue, exploration missions and other scenarios w here cellular infrastructure is unavailable.
\end{abstract}

Key words: Ad hoc network, hop count, link stability, residual energy, route-repair

\section{INTRODUCTION}

A mobile ad hoc network is an autonomous system made up of mobile nodes communicating through wireless links without any static infrastructure. Nodes in this network are self-organizing and rely on each other to relay messages between the nodes. As the nodes are free to move randomly, the network topology may change

Corresponding Author: Anuradha Banerjee, Department of Computer Applications, Kalyani Government Engineering College, Kalyani, Nadia, West Bengal, India 
dynamically. Thus the routing protocol must be equipped with efficient local route-repair schemes to maintain routes in spite of changing network connectivity. Ad hoc networks are very useful in military, disaster mitigation, where emergency rescue is necessary and no static infrastructure in available.

Routing is the most challenging problem in ad hoc networks. Much work has been done in this area and many protocols have been proposed. Of particular interest is the class of on-demand source-initiated routing protocols which set up and maintain routes from a given source to a destination on an "as needed" basis. The on-demand routing protocols like Dynamic Source Routing (DSR) (Johnson and Maltz, 1996) and Ad hoc On-demand Distance Vector routing (AODV) (Perkins and Royer, 1999) rely on global flooding of routerequest packets for route discovery and repair. However, in a large or highly mobile network where links break frequently, frequent route-discovery and repair based on global flooding will render high control overhead and long recovery delay. Thus there is a need for better recovery mechanism that will provide fast, efficient and long-lasting alternative to the broken link. Witness Aided Routing (WAR) (Aron and Gupta, 1999), Associatively Based Routing (ABR) (Toh, 1996; 1999) and Relative Distance Micro-discovery Ad hoc Routing protocol (RDMAR) (Aggelou and Tafazolli, 1999) are mention-worthy routing protocols with their link repair mechanisms embedded. Upon link breakage, WAR (Aron and Gupta, 1999) performs the local recovery by broadcasting data packets with a predefined hop limit. This way it provides fast local recovery but also induces huge overhead as the data packet is broadcasted as a recovery packet (Liu et al., 2003; Youn et al., 2006). ABR (Toh, 1996; 1999) employs an associatively based routing scheme to select the routes likely to be long-lived. However, if a link breakage occurs at a node $n_{a}$, it reports about the route error to the source provided it is closer to the source than the destination. Then the source initiates and endto-end route discovery to find out a suitable route to the same destination. On the other hand, if $\mathrm{n}_{\mathrm{a}}$ is closer to the destination, it broadcasts a route-request with a hop limit equal to the remaining number of hops it was supposed to travel before the link is broken. Only the destination is able to reply to this request. If this succeeds then the route is remedied and no error is reported. Otherwise, a route error is reported to the predecessor $n_{b}$ of node $n_{a}$ in the communication path and $n_{b}$ repeats the process. The recursive process incurs heavy control overhead and energy consumption (Liu et al., 2003). RDMAR (Gui and Mahapatra, 2002) employs a similar approach of local repair as ABR. However, the region of the localized route repair is estimated from the history of distance between the current node and the destination using a location prediction model.

Quick Local Repair scheme using adaptive promiscuous mode (QLR) (Youn et al., 2006) and PATCH (Liu et al., 2003) are two mention-worthy local link recovery schemes. In QLR, after noticing disconnection of a link from $n_{a}$ to $n_{b}, n_{a}$ broadcasts HELP message within its radio-range. Among the nodes that receive this message, those who know about the successor of $\mathrm{n}_{\mathrm{b}}$, reply with an APPROVAL message. After receiving the first APPROVAL message, $\mathrm{n}_{\mathrm{a}}$ and the successor of $\mathrm{n}_{\mathrm{b}}$ accordingly change their own routing tables and the local repair is over (Youn et al., 2006). On the other hand, if no such APPROVAL message arrives at $n_{a}$, route error is reported to the source of the communication and a new route discovery session is initiated by the source. $\mathrm{PATCH}$, on the other hand, is based on the expectation that if a direct link from $n_{a}$ to $n_{b}$ breaks off, there should exist some 2-hop indirect route from $n_{a}$ to $n_{b}$. In order to discover that 2 hop route, from $n_{a}$ broadcasts a routerequest, specifying $\mathrm{n}_{\mathrm{b}}$ as the destination, with limited time-to-live (sufficient for 2 hops). If no route-reply is received from $n_{b}$ within a predefined time interval, $n_{a}$ reports route error to the source receiving which the source initiates a new route discovery to the destination.

In LSNEC, as soon as disconnection of a link from $\mathrm{n}_{\mathrm{a}}$ to $\mathrm{n}_{\mathrm{b}}$ is detected, $\mathrm{n}_{\mathrm{a}}$ broadcasts a ROUTE-REPAIR message within its radio-range specifying $\mathrm{n}_{b}$ or any successor of $\mathrm{n}_{\mathrm{b}}$ in the broken route, as a desirable destination. If any node residing within the radio-range of $n_{\mathrm{a}}$ has an already established single/multi-hop path to any one of those destinations mentioned in the ROUTEREPAIR message, it sends a REPAIR-ACK message back to $n_{a}$. Among the available options, the optimal path is chosen depending upon the residual energy of the nodes and stability of their links with their predecessors in that path. The more the presence of lively nodes and velocity immune links in a communication path, the less frequent are the phenomena of link breakages for them. Hence, in LSNEC, the cost of link repair is much lesser and much time is saved during delivery of data packets. Simulation results strongly support the performance effectiveness of our present scheme.

Relevant observations and definitions: The following practical observations in respect of behavior of ad hoc networks, influenced design of the LSNEC local link repair scheme: 
- According to the study of discharge curve of batteries heavily used in ad hoc networks, at least $40 \%$ of initial battery charge is required by any node to remain in operable condition; $40-60 \%$ of the same is just satisfactory, $60-80 \%$ of it is good whereas the next higher range (80-100\%) indicates that the associated node is very well prepared to take part in communication as far as its energy is concerned (Youn et al., 2006)

- Higher the relative velocity of a node w.r.t. its predecessor in a communication path, lesser is the possibility of survival of the wireless link connecting them. The situation worsens if the nodes are far apart

- If the wireless link between a node and its predecessor in a communication path survives for a long time (without a break), then it has high chance of survival in near future

- If in a communication session, link breakage occurs near the end of the session, then it indicates that most of the data packets have already been delivered to the destination and for delivering the rest we do not really need a very stable route. On the other hand, if most of the data packets are yet to be delivered, then we should be stricter as far as stability of links are concerned, during the process of finding a suitable single/multi-hop alternative to the broken link

Based on the above mentioned observations, we introduce the following terms that will be useful for illustration of the LSNEC scheme.

Residual energy quotient: The residual energy quotient $\alpha 1_{i}(t)$ of a node $n_{i}$ at time $t$ is defined as:

$\alpha 1_{\mathrm{i}}(\mathrm{t})=1-\mathrm{e}_{\mathrm{i}}(\mathrm{t}) / \mathrm{E}_{\mathrm{i}}$

where, $\mathrm{e}_{\mathrm{i}}(\mathrm{t})$ and $\mathrm{E}_{\mathrm{i}}$ indicate the consumed battery power at time $t$ and maximum or initial battery capacity of $n_{i}$, respectively. It may be noted from the formulation in (1) that $0 \leq \alpha 1_{i}(t) \leq 1$. Values close to 1 enhance capability of $n_{i}$ as a router.

Predictive energy quotient: The predictive energy quotient $\alpha z_{\mathrm{i}}(\mathrm{s}, \mathrm{d}, \mathrm{t})$ tries to predict at time $\mathrm{t}$, the residual energy quotient of node $n_{i}\left(n_{i}\right.$ is a router in the present communication path from source $n_{s}$ to destination $n_{d}$ ) at the end of the present communication session between $\mathrm{n}_{\mathrm{s}}$ and $\mathrm{n}_{\mathrm{d}}$. The computation takes into account the rate of depletion of energy in node $n_{i}$ and the time to deliver a packet from source $n_{s}$ to destination $n_{d}$. Please assume the followings:
- $\mathrm{n}_{\mathrm{i}}$ started operating in the network at time $t_{\mathrm{i}}$ with battery capacity $\mathrm{E}_{\mathrm{i}}$

- $\mathrm{n}_{\mathrm{i}}$ consumed $\mathrm{e}_{\mathrm{i}}(\mathrm{t})$ battery power till time $\mathrm{t}$

- data packets started to be delivered from $n_{s}$ to $n_{d}$ in the present communication session at time $\mathrm{t} 1_{\mathrm{s}, \mathrm{d}}$

- $\pi_{\mathrm{s}, \mathrm{d}}$ packets are to be delivered between $\mathrm{n}_{\mathrm{s}}$ to $\mathrm{n}_{\mathrm{d}}$ in the present communication session among which only $\theta_{\mathrm{s}, \mathrm{d}}(\mathrm{t})\left(\theta_{\mathrm{s}, \mathrm{d}}(\mathrm{t}) \leq \pi_{\mathrm{s}, \mathrm{d}}\right)$ packets could be delivered till current time $\mathrm{t}$

$\theta_{\mathrm{s}, \mathrm{d}}(\mathrm{t})$ number of data packets were delivered from $\mathrm{n}_{\mathrm{s}}$ to $\mathrm{n}_{\mathrm{d}}$ in the present communication session in the time interval $\left(\mathrm{t}-\mathrm{t} 1_{\mathrm{s}, \mathrm{d}}\right)$. Approximate time $\gamma_{\mathrm{s}, \mathrm{d}}(\mathrm{t})$ required after time $t$ for completion of the mentioned communication from $n_{s}$ to $n_{d}$, i.e., for delivering the remaining $\left(\pi_{s, d}-\theta_{s, d}(t)\right)$ number of data packets, is given by:

$\gamma_{\mathrm{s}, \mathrm{d}}(\mathrm{t})=\left(\mathrm{t}-\mathrm{t} 1_{\mathrm{s}, \mathrm{d}}\right)\left(\pi_{\mathrm{s}, \mathrm{d}}-\theta_{\mathrm{s}, \mathrm{d}}(\mathrm{t})\right) / \theta_{\mathrm{s}, \mathrm{d}}(\mathrm{t})$

Rate of energy depletion of $n_{i}$ at time $t$ is $e_{i}(t) /\left(t-t_{i}\right)$. Hence, energy consumed by $n_{i}$ till time $\left(t+\gamma_{s, d}(t)\right)$ i.e., $e_{i}\left(t+\gamma_{s, d}(t)\right)$ is expressed as:

$\mathrm{e}_{\mathrm{i}}\left(\mathrm{t}+\gamma_{\mathrm{s}, \mathrm{d}}(\mathrm{t})\right)=\mathrm{e}_{\mathrm{i}}(\mathrm{t})\left(\mathrm{t}+\gamma_{\mathrm{s}, \mathrm{d}}(\mathrm{t})-\mathrm{t}_{\mathrm{i}}\right) /\left(\mathrm{t}-\mathrm{t}_{\mathrm{i}}\right)$

So, $\alpha 2_{\mathrm{i}}(\mathrm{s}, \mathrm{d}, \mathrm{t})=1-\mathrm{e}_{\mathrm{i}}\left(\mathrm{t}+\gamma_{\mathrm{s}, \mathrm{d}}(\mathrm{t})\right) / \mathrm{E}_{\mathrm{i}}$

$\alpha 2_{\mathrm{i}}(\mathrm{s}, \mathrm{d}, \mathrm{t})$ also lies between 0 and 1 .

Lively node: A node $n_{i}$ is termed as lively at time $t$ is provided its remaining battery capacity at that time is at least good i.e., $\alpha 1_{\mathrm{i}}(\mathrm{t}) \geq 0.6$.

Fighter node: A node $n_{i}$ is termed as fighter at time $t$ is provided its predictive energy quotient is between 0.4 and 0.5 .

Fighter route: A route is termed as a fighter provided all its nodes are fighters.

Lively route: A fighter route will be called lively provided all of its nodes are lively.

Minimum communication delay in a multi-hop path: Since the minimum length of a multi-hop path in an ad hoc network, is 2 , minimum delay $\Gamma_{\min }$ for multihop communication is given by:

$\Gamma_{\min }=2 \mathrm{R}_{\min } / \sigma$

Where:

$\sigma=$ Speed of the wireless signal

$\mathrm{R}_{\min }=$ The minimum available radio-range in the network 
Maximum communication delay in a multi-hop path: Assuming $\mathrm{H}$ to be the maximum allowable hop count in the network, maximum number of routers in a communication path is (H-1). If $\tau$ denotes the upper limit of waiting time of that packet in message queue of any node and $R_{\max }$ denotes the maximum available radio-range in the network, maximum delay $\Gamma_{\max }$ for multi-hop communication is given by:

$\Gamma_{\max }=\mathrm{H} \mathrm{R}_{\max } / \sigma+(\mathrm{H}-1) \tau$

In the worst case delay or maximum delay situation, a packet has to traverse the maximum available number of hops i.e., $\mathrm{H}$ with length of each hop being the maximum possible i.e., $\mathrm{R}_{\max }$. Hence the total distance traversed by the wireless signal in its worst case journey from source to destination is $\mathrm{HR}_{\max }$. The signal velocity is $\sigma$ i.e., a packet can traverse $\sigma$ unit distance in unit time. Hence the time required to travel the distance of $\mathrm{HR}_{\max }$, is $\left(\mathrm{HR}_{\max } / \sigma\right)$. This is the upper limit of traveling time for a packet. Also the waiting time in routers are involved in worst case. Maximum age of a packet in message queue of a router is assumed to be $\tau$ and (H-1) is the highest possible number of routers in a path. So, the upper limit of waiting time of a message throughout its journey from source to destination is $(H-1) \tau$. The maximum delay $\Gamma_{\max }$ for multi-hop communication is actually the sum total of the upper limits of the above-mentioned traveling time and waiting time for a packet.

Link stability: Stability $\beta_{\mathrm{ij}}(\mathrm{t})$ of the link between the nodes $n_{j}$ and its predecessor $n_{i}$ in a communication path, is defined in (7) where $n_{j}$ has been continuously residing within neighborhood of $n_{i}$ from $\left(t-\varpi_{i j}(t)\right)$ to current time $\mathrm{t}$ :

$$
\beta_{\mathrm{ij}}(\mathrm{t})=\left\{\begin{array}{cl}
0 & \text { if } \varpi_{\mathrm{ij}}(\mathrm{t}) \leq \Gamma_{\text {min }} \\
1 & \text { if } \varpi_{\mathrm{ij}}(\mathrm{t}) \leq \Gamma_{\text {max }} \\
\left(\frac{\left(\varpi_{\mathrm{ij}}(\mathrm{t})-\Gamma_{\text {min }}\right)}{\Gamma_{\text {max }}-\Gamma_{\text {min }}}\right) & \mathrm{f} 1_{\mathrm{ij}}(\mathrm{t}) \mathrm{f} 2_{\mathrm{ij}}(\mathrm{t}) \text { otherwise }
\end{array}\right.
$$

Where:

$$
\mathrm{f1} 1_{\mathrm{ij}}(\mathrm{t})=\left\{1-\left(\left|\mathrm{v}_{\mathrm{i}}(\mathrm{t})-\mathrm{v}_{\mathrm{j}}(\mathrm{t})\right|+1\right)^{-1}\right\}
$$

and:

$$
\mathrm{f} 2_{\mathrm{ij}}(\mathrm{t})=\left\{1-\mathrm{d}_{\mathrm{ij}}(\mathrm{t}) /\left(\mathrm{R}_{\mathrm{i}}+1\right)\right\}
$$

In the above formulation, $v_{i}(t)$ specifies velocity of node $n_{i}$ at time t. $d_{i j}(t)$ and $R_{i}$ signify the distance between $n_{i}$ and $n_{j}$ at time $t$ and radio range of $n_{i}$, respectively. All other symbols carry their usual meaning. The situation $\varpi_{\mathrm{ij}}(\mathrm{t}) \leq \Gamma_{\min }$, indicates that either $n_{j}$ is completely new as a neighbor to $n_{i}$ or $n_{j}$ did not steadily reside within the neighborhood of $n_{i}$ even for a time interval so small as $\Gamma_{\min }$. Hence the link stability is negligible, denoted by 0 . On the other hand, if $\varpi_{\mathrm{ij}}(\mathrm{t})>\Gamma_{\max }$, it indicates that $\mathrm{n}_{\mathrm{j}}$ has been continuously residing within the neighborhood of $n_{i}$ for more than the time span that may be required at most, for a message to traverse from its source to destination. In this situation the stability is 1 . Otherwise, the ratio $\left(\varpi_{\mathrm{ij}}(\mathrm{t})\right.$ $\left.\Gamma_{\min }\right) /\left(\Gamma_{\max }-\Gamma_{\min }\right)$ is used to predict future of the neighborhood relation between $n_{i}$ and $n_{j}$ based on its history so far. If $\varpi_{\mathrm{ij}}(\mathrm{t})$ is close to $\Gamma_{\text {min }}$, the ratio $\left(\varpi_{\mathrm{ij}}(\mathrm{t})\right.$ $\left.\Gamma_{\min }\right) /\left(\Gamma_{\max }-\Gamma_{\min }\right)$ takes a small fractional value. Similarly, it is evident from (8) that as $\varpi_{\mathrm{ij}}(\mathrm{t})$ approaches $\Gamma_{\max }$, value of the above mentioned ratio proceeds towards 1.

Relative velocity of $n_{i}$ w.r.t. $n_{j}$ at time $t$ is given by $\left(\mathrm{v}_{\mathrm{i}}(\mathrm{t})-\mathrm{v}_{\mathrm{j}}(\mathrm{t})\right)$. Its effect on $\beta_{\mathrm{ij}}(\mathrm{t})$ is modeled as $\mathrm{fl}_{\mathrm{ij}}(\mathrm{t})$. Please note that $f 1_{\mathrm{ij}}(\mathrm{t})$ always takes a fractional value between 0 and 1 , even when $v_{i}(t)=v_{j}(t)$. As the magnitude of relative velocity of $n_{i}$ w.r.t. $n_{j}$ at time $t$ increases, it leads to the decrease in value of $f 1_{\mathrm{ij}}(\mathrm{t})$, which in turn, contributes to increase the link stability.

$\mathrm{f} 2_{\mathrm{ij}}(\mathrm{t})$ expresses the dependence of $\beta_{\mathrm{ij}}(\mathrm{t})$ on the distance between the nodes $n_{i}$ and $n_{j}$ at time t. Since $n_{i}$ is the predecessor of $n_{j}$ at time $t, n_{j}$ must be within the transmission range (or radio-range) of $n_{i}$ at that time. Since $R_{i}$ denotes the radio-range of $n_{i}$, upper limit of the distance $d_{i j}(t)$ between $n_{i}$ and $n_{j}$ at time $t$ is $R_{i}$. As per the expression of $\mathrm{f} 2_{\mathrm{ij}}(\mathrm{t})$ in (10), it also acquires a fractional value between 0 and 1 . As $d_{i j}(t)$ increases, $\mathrm{f}_{2 \mathrm{ij}}(\mathrm{t})$ decreases enhancing the link stability. Note that, $\beta_{\mathrm{ij}}(\mathrm{t})$ always ranges between 0 and 1 .

Residual packet load ratio: Let, in a communication session from source $n_{s}$ to destination $n_{d}, \pi_{s, d}$ packets were to be delivered among which only $\theta_{\mathrm{s}, \mathrm{d}}(\mathrm{t})\left(\theta_{\mathrm{s}, \mathrm{d}}(\mathrm{t})\right.$ $\leq \pi_{\mathrm{s}, \mathrm{d}}$ ) could be delivered till time t. Then the residual packet load ratio $\xi_{s, \mathrm{~d}}(\mathrm{t})$ is given by:

$\xi_{\mathrm{s}, \mathrm{d}}(\mathrm{t})=\theta_{\mathrm{s}, \mathrm{d}}(\mathrm{t}) / \pi_{\mathrm{s}, \mathrm{d}}$

From (10) it is evident that $\xi_{\mathrm{s}, \mathrm{d}}(\mathrm{t})$ lies between 0 and 1.

Stable link: A link will be called stable provided its stability is more than the residual packet load ratio of the communication session at that time. 
Stable route: A multi-hop route will be called stable provided all of its links are stable.

Detailed description of LSNEC: Below we illustrate the local route-repair mechanism of LSNEC based on an established communication path $\mathrm{C}$ from source $\mathrm{n}_{\mathrm{s}}$ to destination $\mathrm{n}_{\mathrm{d}}$ s.t.:

$$
\text { C: } \mathrm{n}_{\mathrm{s}} \rightarrow \mathrm{n}_{\mathrm{i}} \rightarrow \mathrm{n}_{\mathrm{i}+1} \rightarrow \mathrm{n}_{\mathrm{i}+2} \rightarrow \mathrm{n}_{\mathrm{i}+3} \mathrm{v} \ldots \mathrm{n}_{\mathrm{i}+\mathrm{k}} \rightarrow \mathrm{n}_{\mathrm{d}}
$$

Please assume that the link from node $n_{i+1}$ to $n_{i+2}$ is broken. In order to repair the route according to LSNEC, $\mathrm{n}_{i+1}$ broadcasts a ROUTE-REPAIR message within its radio-range specifying $n_{i+2}, n_{i+3}, \ldots n_{i+k}$ and $n_{d}$ as the desirable destinations. After broadcasting the message, $\mathrm{n}_{\mathrm{i}+1}$ waits for a suitable reply till the arrival of the next data packet to it from source $\mathrm{n}_{\mathrm{s}}$. All nodes residing within the transmission range of $n_{i+1}$ will receive the message. If any of them, say $\mathrm{n}_{\mathrm{j}}$, has an already established single/multi-hop communication path to any of those mentioned destinations, it sends a REPAIR-ACK message back to $n_{i+1}$. If $n_{j}$ has an established path to $n_{i+3}$, then this will be termed as a candidate alternative to the broken link from node $n_{i+1}$ to $n_{i+2}$. This candidate alternative begins at node $n_{j}$ and terminates at $n_{i+3}$. Please note that, several other such candidate alternatives may generate from $n_{j}$. Although all of them begins at $n_{j}$, their termination point may be any one of the nodes $n_{i+2}, n_{i+3}, \ldots n_{i+k}$ or $n_{d}$. Corresponding to each of those candidate alternatives to the broken link, the following information are propagated to $\mathrm{n}_{\mathrm{i}+1}$ embedded in the REPAIR-ACK message:

(a) Identification number of the node at which the candidate alternative terminates (any one of $n_{i+2}$, $n_{i+3}, \ldots n_{i+k}$ or $\left.n_{d}\right)$

(b) Identification numbers of the routers present in the path, in proper sequence from $n_{i+1}$ to the specific destination mentioned in (a)

(c) Geographical positions of the nodes mentioned in (b) in terms of latitude and longitude, in same sequence as (b)

(d) Radio-ranges of the nodes mentioned in (b) in the same sequence as in (b) and (c)

(e) Residual energy quotients of the nodes mentioned in (b) in the same sequence as in (b), (c) and (d)

(f) Stability of the links between the consecutive nodes appearing in the sequence specified in (b)

Among all the REPAIR-ACK messages that arrive at $n_{i+1}$ till the arrival of next data packet to it from $n_{s}$, $\mathrm{n}_{\mathrm{i}+1}$ searches for at least one fighter route. If no fighter route is obtained, $\mathrm{n}_{i+1}$ broadcasts a route-request packet to discover a route to $n_{d}$ with maximum allowable number of hops being equal to $\left(H-h_{i+1, s}\right)$ where $h_{i+1, s}$ is the number of hops in the path from $n_{s}$ to $n_{i+1}$.

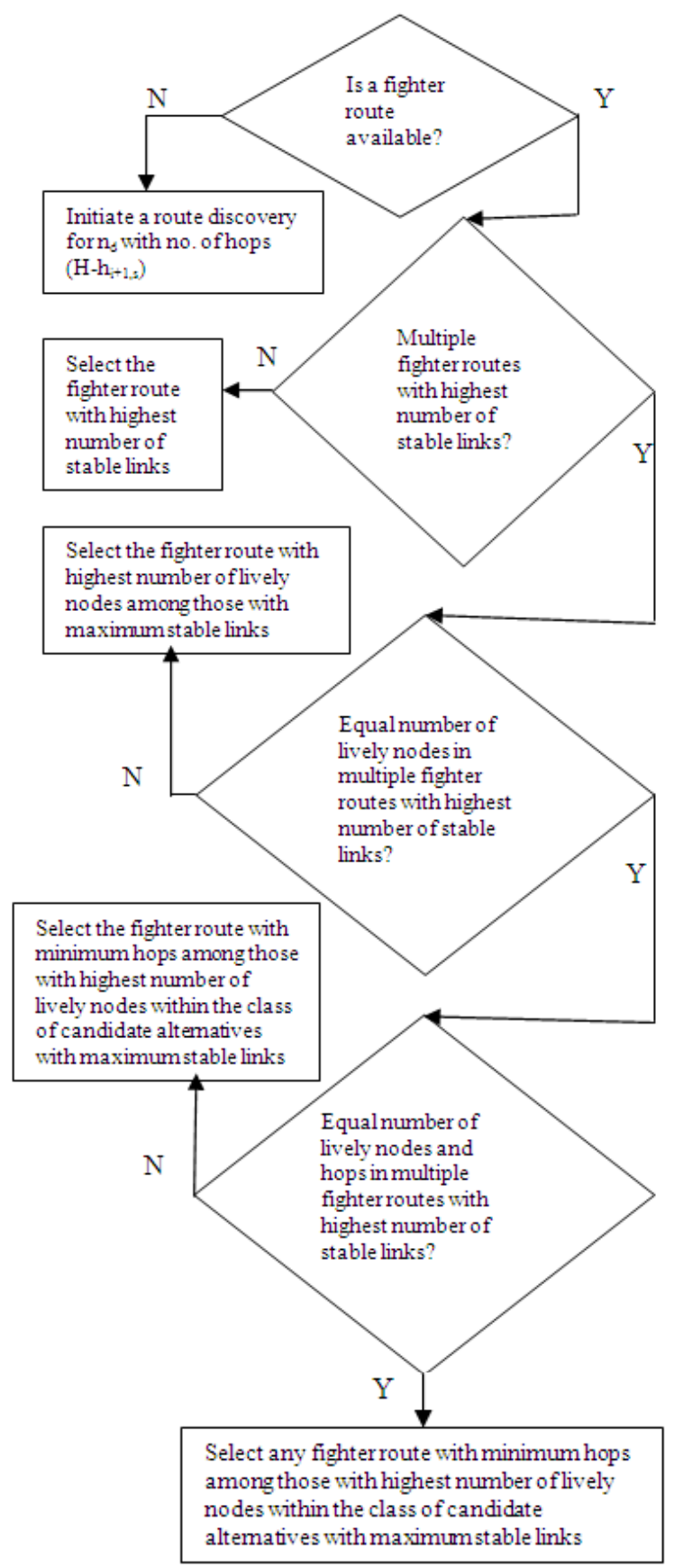

Fig. 1: Demonstration of LSNEC scheme followed by $n_{i+1}$ 
Table 1: Simulation environment

\begin{tabular}{ll}
\hline Mobility pattern & Random way point, random walk \\
\hline Traffic & Constant bit rate \\
Transmission range & $50 \mathrm{~m}$ \\
Mobility & Pause time $10 \mathrm{sec}$, speed $0-35 \mathrm{~m} \mathrm{sec}^{-1}$ \\
Map & $4000 \mathrm{~m} \times 300 \mathrm{~m}, 2000 \mathrm{~m} \times 1600 \mathrm{~m}$ \\
Node number & $40,80,120,160,250,400$ \\
Simulation time & $500 \mathrm{sec}$ \\
\hline
\end{tabular}

If multiple fighter routes are available, $\mathrm{n}_{\mathrm{i}+1}$ chooses the path with maximum number of stable links. In case of availability of multiple such paths, the one with the higher number of lively nodes among them is elected as optimal. In case of presence of equal number of lively nodes in the fighter candidate alternatives with maximum link stability, the one with minimum number of hops among them, is elected as the optimal candidate for repairing the broken link between $n_{i+1} \& n_{i+2}$. If the tie continues even after considering the number of hops, any one of the optimally stable and lively candidates, is elected. Figure 1 shows a flowchart to illustrate the procedure of LSNEC followed by $n_{i+1}$.

\section{MATERIALS AND METHODS}

The simulation is carried out on an $800 \mathrm{MHz}$ Pentium IV processor, 40 GB hard disk and Red Hat Linux version 6.2 operating system. The simulator used is ns-2. Detail about the simulation environment appears in Table 1.

\section{RESULTS}

Simulations were carried out using ns-2 simulator which is a well known packet level simulator, to evaluate the proposed local recovery mechanism. The original AODV and ABR in ns are extended to include PATCH, QLR and LSNEC. Performance of original AODV is compared with PATCH-embedded AODV (PATCH-AODV), QLR embedded AODV (QLRAODV) and LSNEC embedded AODV (LSNECAODV) in Fig. 2, 4, 6 and 8. Similarly, performance of the routing protocol ABR with its route-repair mechanism, is compared with PATCH-embedded ABR (PATCH+ABR routing protocol-ABR route repair mechanism), QLR embedded ABR (QLR+ABR routing protocol-ABR route repair mechanism) and LSNEC embedded ABR (LSNEC+ABR routing protocol-ABR route repair mechanism) in Fig. 3, 5, 7 and 9.

In our simulations, high mobility is used such that consistent breakages in the routes can be observed. To emphasize the effectiveness of our proposed mechanism, a long map of $4000 \times 300 \mathrm{~m}^{2}$ is used, such that the average route length is generally long.

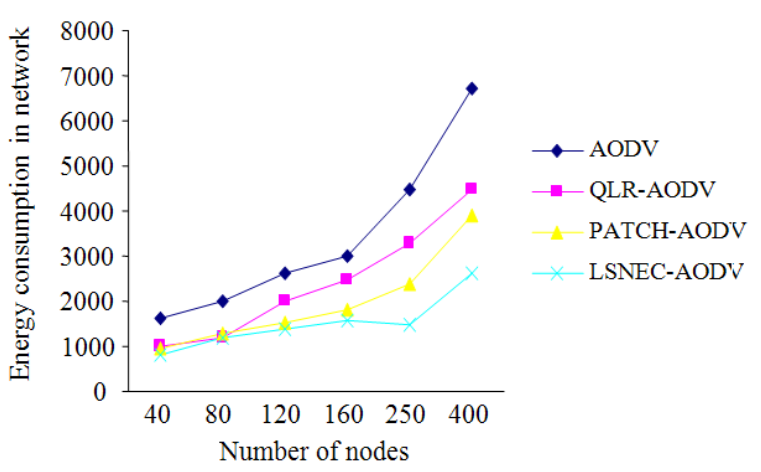

Fig. 2: Energy consumption in AODV compared with its PATCH, QLR and LSNEC embedded versions

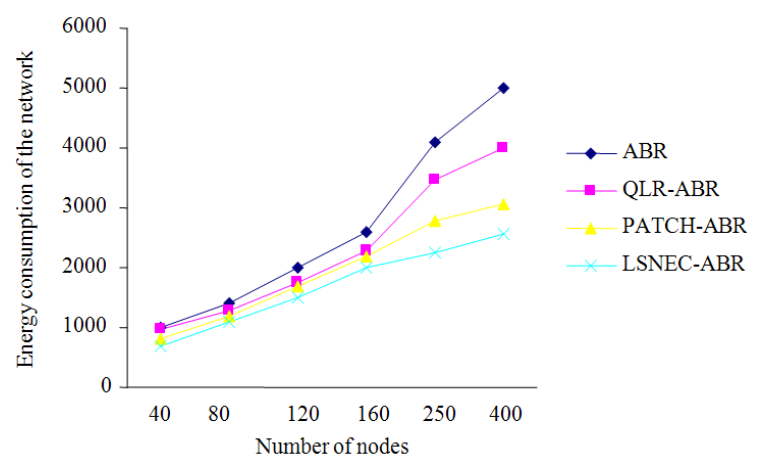

Fig. 3: Energy consumption in ABR compared with its PATCH, QLR and LSNEC embedded version

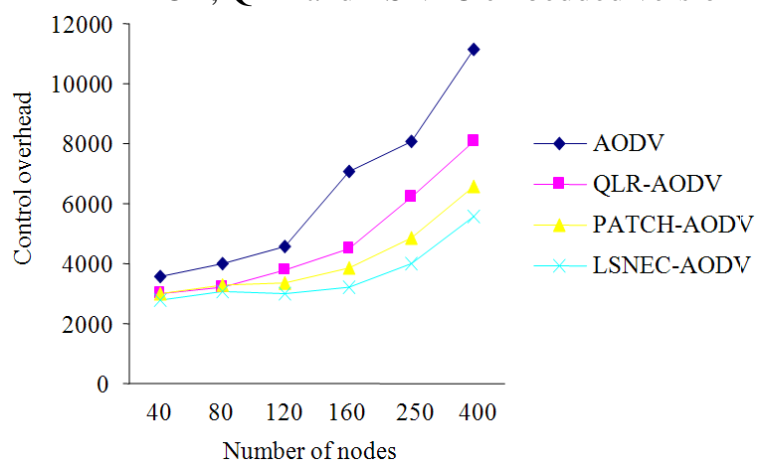

Fig. 4: Control overhead in AODV compared with its PATCH, QLR and LSNEC embedded versions

However, considerable improvements are also seen from simulations run on a broad map of $2000 \mathrm{~m} \times 1600 \mathrm{~m}$. Lastly, simulations were run across various densities. With increasing density, the average degree (number of nodes with transmission range of a node) of the nodes keep increasing and thus the possibility of successful local recovery also increase. 


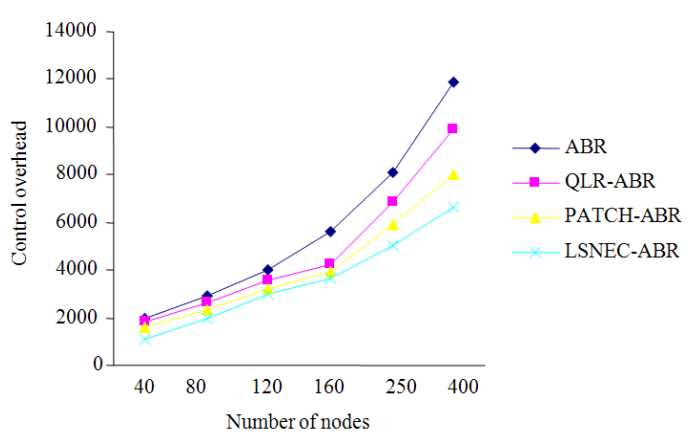

Fig. 5: Control overhead in ABR compared with its PATCH, QLR and LSNEC embedded versions

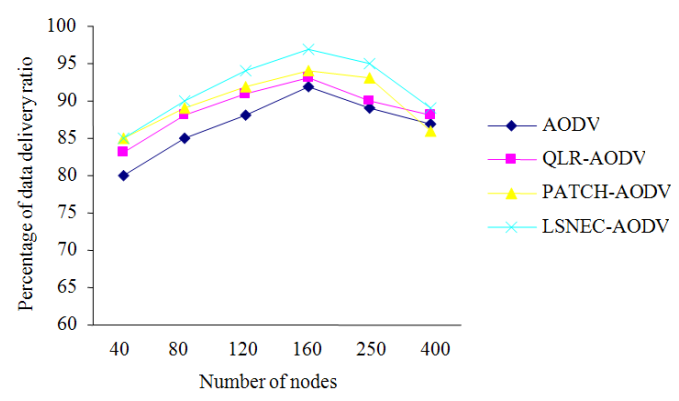

Fig. 6: Percentage of data delivery ratio in AODV compared with its PATCH, QLR and LSNEC embedded versions

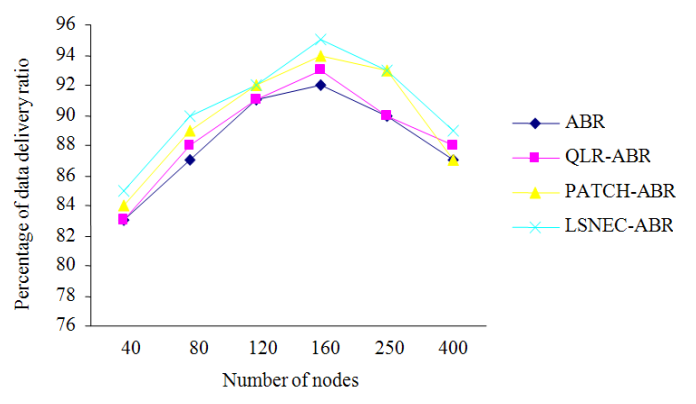

Fig. 7: Percentage of data delivery ratio in $A B R$ compared with its PATCH, QLR and LSNEC embedded versions

Simulation metrics are percentage of data delivery ratio (the number of data packets successfully delivered to their respective destinations/the number of data packets transmitted by various sources), control overhead (total number of control packets injected into the network), energy consumption of the network (summation of consumed energy of all the nodes throughout the simulation period) and average delay in route-recovery per communication session (total recovery delay in all communication sessions/total number of sessions). The delay is expressed in seconds.

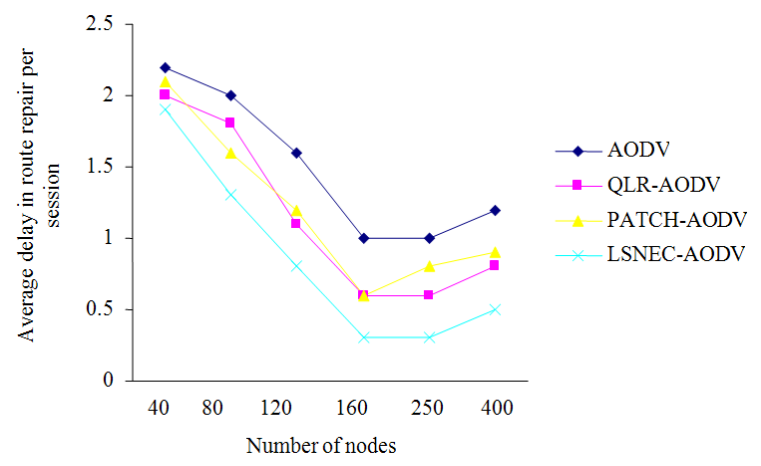

Fig. 8: Average delay in route-repair per session in AODV compared with its PATCH, QLR and LSNEC embedded versions

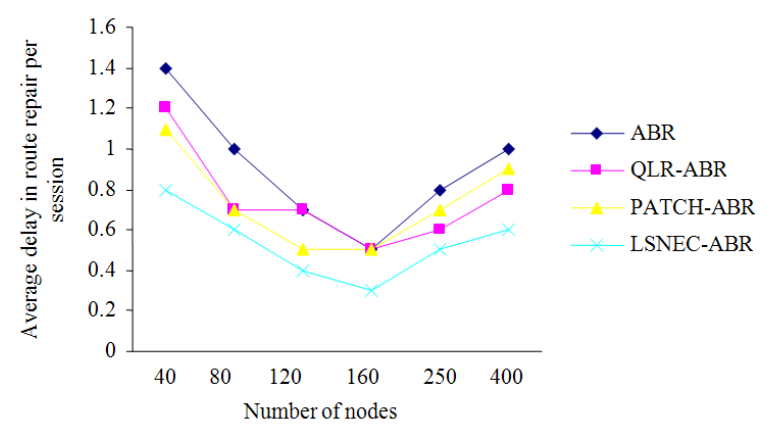

Fig. 9: Average delay in route-repair per session in ABR compared with its PATCH, QLR and LSNEC embedded versions

The results are averaged over 30 sets of simulation results and plotted at $95 \%$ confidence interval.

\section{DISCUSSION}

At low node density in the network, none of the mentioned recovery schemes show much advantage. Especially when the number of nodes is as low as 40, the connectivity of the whole network is not quite good and the problem of partitioning may be severe. Most of the transmission is successful only in small partitions with short route length. In such situation, the local recovery covers most portion of the whole partition already, thus we cannot see obvious control packet saving at low density. However, as the density goes higher, the connectivity of the network becomes higher; transmission with longer route length can be formed at this stage. In such situations, local recovery schemes start to show obvious improvement over end-to-end recovery scheme, as local recovery floods the route repair request in a small region whereas end-to-end recovery floods the entire network. Moreover, unlike 
QLR and PATCH, LSNEC is concerned with stability of links and remaining charge as well as rate of energy depletion of the nodes. Hence, control overhead in LSNEC embedded versions of protocols is much smaller compared to their ordinary version and QLR and PATCH embedded versions. Less control overhead yields less energy consumption and less network congestion as well. Decrease in network congestion greatly reduces the possibility of packet collision. As a result, percentage of successful packet delivery ratio increases significantly. For all the above-mentioned protocols, packet delivery ratio is low when the number of nodes in the network is as low as 40 . Then it starts increasing till the network is saturated with nodes after which the delivery ratio goes down again. The reason is that, initially the network is partitioned and lots of data packets fail to reach the destination due to unavailability of routes. The situation gradually repairs as the node density increases. After that, when the network gets saturated with nodes, collision among the packets destroy some data packets before they arrive at the destination.

As far as delay in route-repair is concerned, LSNEC produces huge improvement compared to ordinary AODV because ordinary AODV always goes for endto-end route discovery instead of local recovery as in LSNEC, QLR and PATCH. After a link breakage, both QLR and PATCH communicate through the first available alternative. On the contrary, LSNEC waits till the arrival of next data packet and chooses the optimal one out of the options available. The optimality criteria consist of remaining energy of nodes, their rate of depletion of energy and stability between consecutive links. Preferring the stable links reduce the possibility of further link breakages in that session.

Table 2: Performance enhancement produced by LSNEC-AODV over ordinary AODV, QLR-AODV and PATCH-AODV

\begin{tabular}{llll}
\hline & $\begin{array}{l}\text { LSNEC- } \\
\text { AODV over } \\
\text { AODV }(\%)\end{array}$ & $\begin{array}{l}\text { LSNEC-AODV } \\
\text { over QLR- } \\
\text { AODV }(\%)\end{array}$ & $\begin{array}{l}\text { LSNEC-AODV } \\
\text { over PATCH- } \\
\text { AODV }(\%)\end{array}$ \\
\hline Performance metric & 21.20 & 18.87 & 18.15 \\
\% of data delivery ratio & 57.00 & 36.31 & 32.70 \\
Control overhead & 54.68 & 37.50 & 31.11 \\
$\begin{array}{l}\text { Energy consumption } \\
\text { Average delay in route- }\end{array}$ & 47.45 & 20.89 & 18.43 \\
repair per session & & & \\
\hline
\end{tabular}

Table 3: Performance enhancement produced by LSNEC-ABR over ordinary ABR, QLR-ABR and PATCH-ABR

\begin{tabular}{lccc}
\multicolumn{4}{c}{ ordinary ABR, QLR-ABR and PATCH-ABR } \\
\hline & $\begin{array}{l}\text { LSNEC- } \\
\text { ABR over }\end{array}$ & $\begin{array}{l}\text { LSNEC-ABR } \\
\text { over QLR }\end{array}$ & $\begin{array}{l}\text { LSNEC-ABR } \\
\text { over PATCH }\end{array}$ \\
Performance metric & ABR $(\%)$ & -ABR $(\%)$ & -ABR $(\%)$ \\
\hline$\%$ of data delivery ratio & 7.40 & 4.29 & 5.00 \\
Control overhead & 29.30 & 12.80 & 10.70 \\
Energy consumption & 28.14 & 12.00 & 11.89 \\
Average delay in route- & 21.90 & 8.19 & 9.84 \\
repair per session & & &
\end{tabular}

Hence the number of occurrences of link breakage in LSNEC embedded protocols is much less than QLR and PATCH embedded versions of those. This results in the reduction of delay in route-repair per session. Also note that, LSNEC-AODV produces huge improvement than QLR-AODV and PATCH-AODV compared to the improvement generated by LSNEC-ABR over QLR$A B R$ and PATCH-ABR. The reason is that, unlike AODV, ABR elects routes based on associatively between consecutive nodes where stability is predicted depending upon history of the neighborhood relation between the nodes. Percentage of performance enhancement produced by LSNEC embedded versions of the protocols AODV and ABR compared to their ordinary versions and QLR as well as PATCH embedded versions are shown in Table 2 and 3.

\section{CONCLUSION}

In the present article we propose a Link Stability and Node Energy Conscious (LSNEC) local recovery mechanism that tries to discover a stable and energetic alternative to broken links. This reduces the possibility of further breakage in the repaired portion of the route. On the whole, vast improvements have been seen in simulation results. The savings in terms of node energy also prevents the network from being partitioned and thereby increasing the data packet delivery ratio. On contrary to other local recovery protocols, LSNEC does not choose the first available alternative, but waits for arrival of next data packet from source; examines all the alternatives arrived by that time and then elects the optimal among them. This greatly reduces the control overhead, delay in route repair and saves network energy significantly. Future work consists of studying more extensively the performance of LSNEC with the other ad hoc network routing protocols with other different sets of parameters.

\section{REFERENCES}

Aggelou, G. and R. Tafazolli, 1999. RDMAR: A bandwidth-efficient routing protocol for mobile ad hoc networks. Proceedings of the 2nd ACM International Workshop on Wireless Mobile Multimedia, Aug. 20-20, ACM Press, Seattle, Washington, United States pp: 26-33. DOI: $10.1145 / 313256.313272$

Aron, I.D. and S.K.S. Gupta, 1999. A witness-aided routing protocol for mobile ad hoc networks with unidirectional links. Proceeding of the 1st International Conference on Mobile Data Access, Dec. 16-17, Springer-Verlag, London, UK., pp: 24-33. http://portal.acm.org/citation.cfm?id=746196 
Gui, C. and P. Mahapatra, 2002. A self-healing and optimizing routing technique for ad hoc networks. National Science Foundation. http://www.cs.ucdavis.edu/research/techreports/2002/CSE-2002-23.pdf

Johnson, D.B. and D.A. Maltz, 1996. Dynamic source routing protocol for mobile ad hoc wireless networks. Mobile Comput., 353: 153-181. DOI: 10.1007/978-0-585-29603-6_5

Liu, G., K.J. Wong, B.S. Lee, B.C. Seet and C.H. Foh et al., 2003. PATCH: A novel local recovery mechanism for mobile ad hoc networks. Proceeding of the IEEE 58th Vehicular Technology Conference, Oct. 6-9, IEEE Xplore Press, USA., pp: 2995-2999. DOI: 10.1109/VETECF.2003.1286173

Perkins, C.E. and E.M. Royer, 1999. Ad-hoc ondemand distance vectored routing. Proceeding of the 2nd IEEE Workshop on Mobile Computing Systems and Applications, Feb. 25-26, IEEE Xplore Press, New Orleans, LA., USA., pp: 90-100. DOI: 10.1109/MCSA.1999.749281
Toh, C.K., 1996. A novel distributed routing protocol to support ad-hoc mobile computing. Proceeding of the IEEE International Phoenix Conference on Computers and Communications, Phoenix, USA., Mar. 27-29, Scottsdale, IEEE Xplore Press, AZ., USA., $\quad$ pp: 480-486. DOI: 10.1109/PCCC.1996.493675

Toh, C.K., 1999. Long-lived ad hoc routing based on the concept of associatively. Internet draft IETF. http://tools.ietf.org/html/draft-ietf-manet-longlivedadhoc-routing-00

Youn, J.S., J.H. Lee, D.H. Sung and C.H. Kang, 2006. Quick local repair using adaptive promiscuous mode in mobile ad hoc networks. J. Networks, 1: $1-11$. http://www.academypublisher.com/jnw/vol01/no01 /jnw01010111.pdf 